\title{
Oriented attachment of nanoparticles to form micrometer-sized nanosheets/nanobelts by topotactic reaction on rigid/flexible substrates with improved electronic properties
}

\author{
Hong-mei Qian ${ }^{1}$, Qian Zhao ${ }^{1}$, Bao-song Dai ${ }^{1}$, Li-jie Guo ${ }^{2}$, Jin-xing Zhang ${ }^{2}$, Jia-jia Liu ${ }^{1}$, Jia-tao Zhang ${ }^{1}$ \\ and $\mathrm{He}$-sun $\mathrm{Zhu}^{1}$
}

\begin{abstract}
We report a topotactic reaction strategy to achieve the oriented attachment $(O A)$ of colloidal metal chalcogenide quantum dots into micrometer-sized nanosheets and nanobelts (up to 6-7 $\mu \mathrm{m}$ ) on both mechanically rigid and flexible substrates. The nonstoichiometric composition, crystallization and Ag doping were controlled. The strong surface adsorption of cations and thiol ligands facilitated micrometer-scale three-dimensional OA. The cations induced the formation of electrostatic forces, cation passivation on the nanosheet surface and overlap packing of the nanosheets, enabling good contact with the substrates and improved electron transport without severe obstruction of organic insulating barriers. The observation of weak anti-localization phenomena and Hall effect sensitivity (up to $188 \%$ ) of non-stoichiometric $\mathrm{Ag}_{2-\delta} \mathrm{Te}$ nanosheet films as well as the improved I-V and photoresponse properties of Ag-doped CdX nanosheet films confirm efficient electron transport. The stable I-V properties of these nanosheet films on flexible substrates, even under bending forces, testify to their potential in flexible device applications. NPG Asia Materials (2015) 7, e152; doi:10.1038/am.2014.120; published online 9 January 2015
\end{abstract}

\section{INTRODUCTION}

In colloidal-quantum-dot (CQD) optoelectronics, the long-chained capping ligands between CQDs produce insulating barriers that interfere with efficient carrier transport when processed into films. ${ }^{1}$ For device applications, the oriented assembly of CQDs and a decrease in these organic ligand-induced carrier barriers are necessary to achieve improved electronic transport. ${ }^{1-3}$ The controllable bottomup assembly of CQDs into films without severe side effects from organic insulating barriers is the key step to achieving improved electronic transport. The oriented attachment (OA) growth mechanism developed by Penn and Banfield et al. ${ }^{4,5}$ has been widely used to assemble CQD building blocks into free-floating nanosheets. ${ }^{6-11}$ However, these colloidal nanosheets generally still have strong organic ligands on the surface that limit the charge carrier transfer/transport in their film applications. Thus, large-scale overgrowth of these sheets with a given pattern onto rigid/flexible substrates to achieve improved electronic transport and contact with the substrates is ideal for exploring their fundamental physics and various solid-state optoelectronics, such as low-cost photovoltaic and on-chip nanoelectronic devices. ${ }^{1,12-16}$

Metal chalcogenide (MX) CQDs have attracted considerable attention owing to their shape- and size-dependent optical and electronic properties and their potential applications in various areas, including optics, electronics and catalysis. ${ }^{17-21}$ For practical optoelectronic device applications, it is useful to assemble MX CQDs into large crystalline nanosheets and then perform the wafer-scale assembly of nanosheets on diverse substrates without organic insulating barriers. ${ }^{22,23}$ Here, we demonstrate a novel low-temperature topotactic reaction with the 3-D OA of CQDs on both rigid and flexible substrates to obtain micrometer-sized MX nanosheets with precise control of doping, crystallization and non-stoichiometric composition. The close packing of these nanosheets with highly preferred orientation and good contact leads to improved electronic properties, such as magnetotransport and optoelectronic response properties in the bulkassembled film.

\section{EXPERIMENTAL PROCEDURE}

Chemicals and materials

All chemicals were used as received without further processing. The conductive indium tin oxide glass (ITO glass) substrates $(<10 \mathrm{ohm}$ per sq) and the flexible polyethylene terephthalate (PET) ITO film substrates ( $45 \pm 5 \mathrm{ohm}$ per sq) were purchased from Zhuhai Kaivo Electronic Components, Guangdong, China. The ITO glass substrates were cleaned by the 'Piranha solution' method, and the flexible PET-ITO substrates were pretreated with (3-aminopropyl) trimethoxysilane. The $p$-type single-crystalline (100) silicon wafers with resistivity of 0.03 -

\footnotetext{
${ }^{1}$ Research Center of Materials Science, School of Materials Science \& Engineering, Beijing Institute of Technology, Beijing, PR China and ${ }^{2}$ Department of Physics, Beijing Normal University, Beiiing, PR China

Correspondence: Professor J-t Zhang, Research Center of Materials Science, School of Materials Science\& Engineering, Beijing Institute of Technology, No.5 South Street of Zhongguancun, Haidian district, Beijing 100081, PR China.

E-mail: zhangit@bit.edu.cn

Received 18 April 2014; revised 21 September 2014; accepted 29 October 2014
} 
$0.01 \Omega . c m$ were purchased from Semiconductor Research Institute of Tianjin, China. All the substrates were pretreated with $5 \mathrm{moll}^{-1} \mathrm{KOH}$ solution to ensure a negatively charged surface.

\section{Preparation of micrometer-sized amorphous/crystalline $\mathrm{Ag}_{2-\delta} \mathrm{Te}$ nanosheets, Ag-doped quasi-sc CdTe nanosheets on given rigid/flexible substrates and wafer-scale packing}

Amorphous $\mathrm{Ag}_{2-\delta} \mathrm{Te}$ nanosheets were prepared by the topotactic reaction between micrometer-sized Ag nanosheets (thickness $<30 \mathrm{~nm}$, Supplementary Figure S1) on the substrate with an organic Te precursor. ${ }^{24}$ Typically, micrometer-sized Ag nanosheets on a given substrate $(1 \times 1 \mathrm{~cm})$ were immersed in a mixture of $8 \mathrm{ml}$ ethanol, $0.05 \mathrm{ml}$ acetone and $0.005 \mathrm{ml}$ dodecanethiol in a glass vessel at room temperature. A solution of $0.5 \mathrm{ml} \mathrm{Te}$ precursor $(1 \mathrm{mmol} \mathrm{Te}$ powder mixed with $7.5 \mathrm{ml}$ toluene and $7.5 \mathrm{ml}$ trioctylphosphine (TOP), heated at $50^{\circ} \mathrm{C}$ for $30 \mathrm{~min}$ ) was added. The vessel was then sealed and maintained at $25-65^{\circ} \mathrm{C}$ for $4.5 \mathrm{~h}$. The liquid was withdrawn, and the substrate with amorphous/crystalline $\mathrm{Ag}_{2-\delta} \mathrm{Te}$ film was obtained and washed with ethanol. For crystalline $\mathrm{Ag}_{2-\delta} \mathrm{Te}$ nanosheet films, after reaction with the Te precursor at $\sim 25^{\circ} \mathrm{C}$, the film was maintained at $60^{\circ} \mathrm{C}$ for $2 \mathrm{~h}$ in $1 \mathrm{ml}$ of methanol with $0.06 \mathrm{~g} \mathrm{Cd}\left(\mathrm{NO}_{3}\right)_{2} \bullet 4 \mathrm{H}_{2} \mathrm{O}$ to permit the interaction of $\mathrm{Cd}^{2+}$ with the longchained organic ligands.

For the further preparation of CdTe nanosheets, $6 \mathrm{ml}$ toluene, $0.2 \mathrm{ml}$ oleic acid and $0.1 \mathrm{ml}$ oleylamine were added to the vessel to cover the amorphous $\mathrm{Ag}_{2-\delta} \mathrm{Te}$. One millilitre of methanol with $0.06 \mathrm{~g} \mathrm{Cd}\left(\mathrm{NO}_{3}\right)_{2} \bullet 4 \mathrm{H}_{2} \mathrm{O}$ and $0.13 \mathrm{ml}$ TBP was added. The resulting mixture was sealed and maintained at $60^{\circ} \mathrm{C}$ for $2 \mathrm{~h}$ to yield CdTe nanosheets on the substrate. The wafer-scale packing of CdTe nanosheets on the substrates was obtained using the same method, except that the Ag nanosheets were first close packed on the substrate.

\section{Preparation of Ag-doped quasi-sc CdS nanosheets on substrates and their wafer-scale packing}

Amorphous $\mathrm{Ag}_{2-\delta} \mathrm{S}$ nanosheets were prepared by the topotactic reaction between Ag nanosheets (thickness $<30 \mathrm{~nm}$ ) on the substrate and an organic $\mathrm{S}$ precursor at room temperature. ${ }^{24}$ Typically, the Ag nanosheets on the given rigid/flexible substrate $\left(1 \times 1 \mathrm{~cm}^{2}\right)$ were immersed in a mixture of $5 \mathrm{ml}$ ethanol, $0.2 \mathrm{ml}$ acetone and $0.02 \mathrm{ml}$ dodecanethiol in a glass vessel at room temperature. Two milliliters of S precursor ( $2 \mathrm{mmol} \mathrm{S}$ powder mixed with $5 \mathrm{ml}$ oleylamine, $10 \mathrm{ml}$ oleic acid and $15 \mathrm{ml}$ toluene, and heated at $100^{\circ} \mathrm{C}$ for $40 \mathrm{~min}$ ) was added. The resulting mixture was sealed and maintained at $25^{\circ} \mathrm{C}$ for $4.5 \mathrm{~h}$. After drying in air, $6 \mathrm{ml}$ toluene, $0.2 \mathrm{ml}$ oleic acid and $0.1 \mathrm{ml}$ oleylamine were added to the vessel. Then, $1 \mathrm{ml}$ methanol with $0.06 \mathrm{~g} \mathrm{Cd}\left(\mathrm{NO}_{3}\right)_{2}$ and $0.1 \mathrm{ml}$ tributylphosphine (TBP) was added. The resulting mixture was sealed and maintained at $60^{\circ} \mathrm{C}$ for $2 \mathrm{~h}$ to obtain $\mathrm{CdS}$ nanosheets. The hierarchical assembly of CdS nanosheets was obtained similarly except that $\mathrm{Ag}$ nanosheets were first hierarchically assembled on the substrate.

\section{Structure and morphology characterization}

The 3-D and 2-D AFM measurements of CdTe or CdS nanosheets and nanobelts on the substrate were obtained using a Digital Instruments Nanoscope-V Multimode AFM (Dimension 3100, Bruker, Santa Barbara, USA). The 2-D images were recorded in tapping mode in air. The AFM tip used was RTESP, made of phosphorus ( $\mathrm{n}$ ) doped $\mathrm{Si}$, and the scan rate was $0.3-0.6 \mathrm{~Hz}$

Low-resolution TEM (JEOL JEM 1200EX working at $100 \mathrm{kV}$ ) and highresolution TEM (FEI Tecnai G2 F20 S-Twin working at $200 \mathrm{kV}$ ) were utilized to characterize the details of the morphology and interfacial lattices. The SAED measurement was performed using a FEI Tecnai G2 F20 S-Twin. The s.e.m. images and energy-dispersive spectrometry (EDS) analysis were obtained using a Hitachi FE-s.e.m. 4800. The phases of the products were determined by XRD on a Bruker D8 Advance X-ray powder diffraction meter with $\mathrm{Cu}$ Ko radiation $(\lambda=1.5418 \AA)$.
I-V curve measurement of single CdTe nanobelts on Si wafers by conductive probe AFM

Conductive probe-AFM (CP-AFM) was used to measure electrical properties with Bruker's Dimension Icon AFM instrument at room temperature in air. For CP-AFM, a conductive silicon probe with $\mathrm{Pt} / \mathrm{Ir}$ layer coating on the surface (STM-PIT, Bruker, tip radius of $\sim 15 \mathrm{~nm}$ ) was used. The current-voltage (I-V) characteristics of single CdTe nanobelts on a Si wafer were measured in the TUNA (Tunneling Atomic Force Microscopy) mode. The conductive tip in contact with the CdTe nanobelt served as the top electrode, and the Si substrate was connected with a conducting wire to become the external electrode. The conductive probe scanned the sample surface in contact mode. After the surface morphology of the sample was obtained, AFM was used to conduct a single point I-V spectrum test. At a certain point of the sample where the tip and the sample surface were in contact, a continuously changing bias voltage was applied between them. The TUNA current was captured by the current amplifier that was connected to the conductive probe. The I-V curves were recorded using the same tip at different positions on CdTe nanobelt.

\section{XPS depth profiling analysis}

The XPS depth profiling (S-probe, Surface Science Instruments, VG with a monochromatic $\mathrm{Al} \mathrm{K \alpha}$-source, 1,486.6 eV) was obtained as follows: the voltage and power of the source were kept constant at $15 \mathrm{KV}$ and $25 \mathrm{~W}$. Sputtering of an area of $2 \times 2 \mathrm{~mm}^{2}$ for 9 min was performed using an $\mathrm{Ar}^{+}$ion gun $(4 \mathrm{keV})$. The XPS spectra were obtained by PHI Quantera II X-ray photoelectron spectroscopy using $\mathrm{Al} \mathrm{K}$-alpha non-monochromatic radiation. The measurement parameters were as follows: light spot size, $100 \mu \mathrm{m}$; power, $100 \mathrm{~W}$; voltage, $20 \mathrm{KV}$. An energy correction was made to account for sample charging based on the carbon $(1 \mathrm{~S})$ peak at $284.8 \mathrm{eV}$. The elemental concentrations are reported relative to carbon, calculated from the XPS spectra based on the area of the characteristic photoelectron peaks after correcting for atomic sensitivity. The fitting analysis was performed by XPS Peak software.

\section{Device fabrication, Hall effect and magnetotransport measurements} of $c-\mathrm{Ag}_{2-\delta} \mathrm{Te}$ nanosheet films

Conductive silver glue was used to prepare Ag electrodes to obtain ohmic contact with samples. For the Hall effect measurements, the position of $\mathrm{Ag}$ electrodes at four corners of a square film was controlled precisely to obtain four sides with the same length. The I-V curves and Hall effect of $1 \times 1 \mathrm{~cm}^{2}$ $c$ - $\mathrm{Ag}_{2-\delta} \mathrm{Te}$ nanosheet films were measured in air by LakeShore VSM 7407 and Keithley 4200 semiconductor parameter analyzers. The magnetotransport measurements were performed using a $9 \mathrm{~T}$ physical properties measurement system (9 T PPMS, Quantum Design) from 1.9 to $400 \mathrm{~K}$ in fields up to $9 \mathrm{~T}$. The five-electrode Hall bar configuration was fabricated for the transport measurements.

\section{I-V, photoconductivity and response characterizations of $\mathrm{CdX}$ films} on conductive PET and ITO substrates

The I-V curves of as-prepared $c$ - $\mathrm{Ag}_{2-\delta} \mathrm{Te}$, quasi-sc $\mathrm{CdTe}$ and quasi-sc $\mathrm{CdS}$ nanosheet films on flexible PET substrates under different bending forces and on rigid ITO substrates were measured by a semiconductor characteristic measurement system (Keithley 4200 SCS and PW-600 probe station) at room temperature in ambient conditions. The PET conductive film was connected by one probe and the other was connected to the top central part during bending of the as-prepared film. The I-V curves of as-prepared CdTe films on glass substrates were also measured in air by LakeShore VSM 7407 and Keithley 4200 semiconductor parameter analysers. The samples were illuminated with a $300 \mathrm{~W}$ Xe lamp (CEL-HXF300/CEL-HXUV300). The photoconductivity and response of the device were measured using a Keithley 2400.

\section{RESULTS AND DISCUSSION}

Structure and morphology of nanosheets and nanobelts

A schematic of the topotactic reaction protocol is outlined in Figure 1. Amorphous non-stoichiometric silver chalcogenide $\left(a-\mathrm{Ag}_{2-\delta} \mathrm{X}\right)$ nanosheets structured by aggregated nanoparticles on rigid/flexible substrates were chosen as starting materials. The $a-\mathrm{Ag}_{2-\delta} \mathrm{X}$ nanosheets 


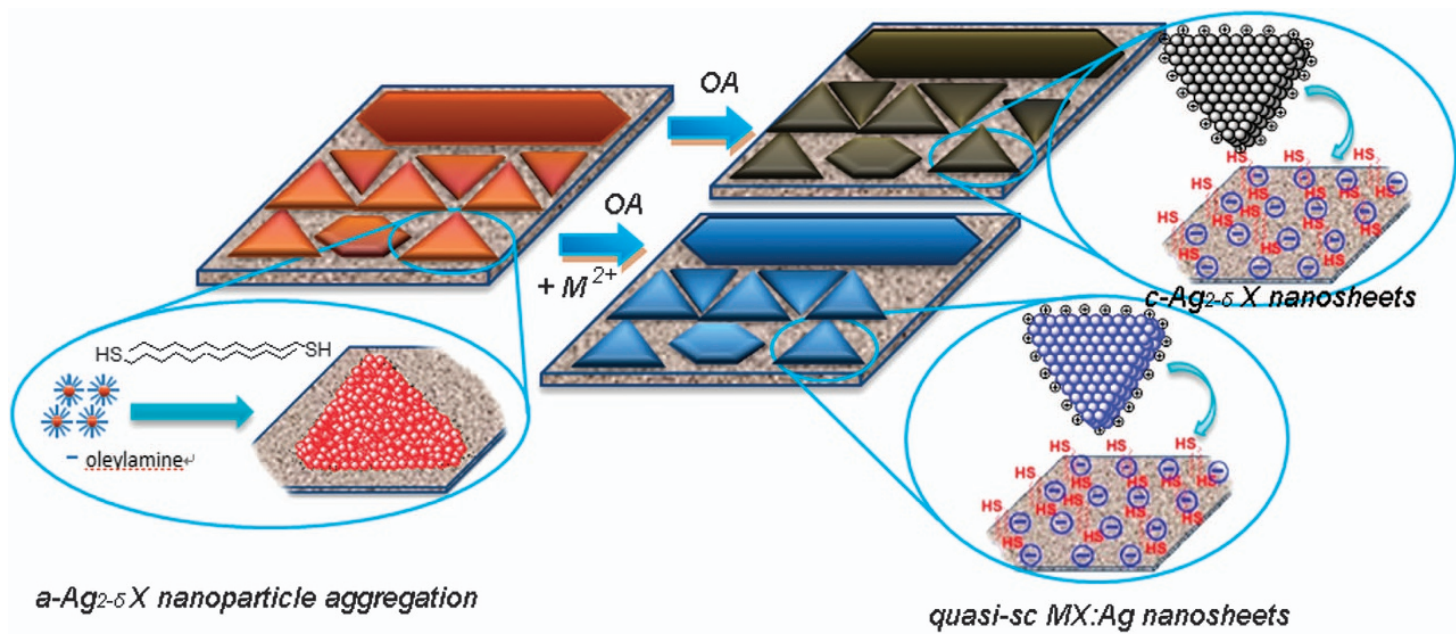

Figure 1 Schematic of $a_{-}-\mathrm{Ag}_{2-\delta} \mathrm{X}$ nanoparticles becoming micrometer-sized $\mathrm{c}-\mathrm{Ag}_{2-\delta} \mathrm{X}$ and quasi-sc Ag-doped $\mathrm{MX}$ nanosheets/nanobelts by topotactic reactioninduced 3-D OA growth on rigid/flexible substrates. Cation-induced electrostatic force, cation and thiol ligand passivation on the nanosheet surface help achieve good contact with the substrates.

were prepared on substrates through the solid-solution interfacial reaction between the $\mathrm{Ag}$ nanosheets and a slight excess of organic $\mathrm{X}$ precursors, such as oleic acid/oleylamine-coordinated sulfur or TOP-coordinated tellurium with moderate soft-acid-base reactivity at room temperature. The amorphous state is necessary for the non-stoichiometric composition of crystalline $\mathrm{Ag}_{2-\delta} \mathrm{X} \quad\left(c-\mathrm{Ag}_{2-\delta} \mathrm{X}\right)$ nanosheets (see the EDS elemental analysis of $a-\mathrm{Ag}_{2-\delta} \mathrm{S}$ and $c-\mathrm{Ag}_{2-}$ ${ }_{\delta} \mathrm{Te}$ in Supplementary Figure S2), cation exchange and Ag doping in $\mathrm{CdX}$ nanosheets. Then, taking advantage of the strong surface adsorption of cations and thiol ligands, the $a-\mathrm{Ag}_{2-\delta} \mathrm{X}$ nanosheets could be converted into $c-\mathrm{Ag}_{2-\delta} \mathrm{X}$ nanosheets in $\mathrm{Cd}^{2+}$ methanol solution at $50-70{ }^{\circ} \mathrm{C}$ through the $3-\mathrm{D}$ OA growth of $\mathrm{Ag}_{2-\delta} \mathrm{X}$ CQDs. Without addition of TBP, the $\mathrm{Cd}^{2+}$ can exchange most weak capping ligands, such as oleic acid, oleylamine and TOP. ${ }^{1}$ Then, it provided electrostatic force and cation passivation on $c-\mathrm{Ag}_{2-\delta} \mathrm{X}$ nanosheets, from which better contact with negative-charged substrate could be obtained. With the addition of TBP, by the interfacial cation exchange reaction between the $a-\mathrm{Ag}_{2-\delta} \mathrm{X}$ nanosheets and $\mathrm{Cd}^{2+}$ ions in methanol solution, ${ }^{24}$ quasi-single-crystalline (quasi-sc) CdX nanosheets could be obtained on rigid/flexible substrates through the 3-D OA of CdX CQDs. The excess of $\mathrm{Cd}^{2+}$ ions also can stabilize the surface of the $\mathrm{CdX}$ nanosheets to give better contact with the substrate.

The morphologies of the CdX nanosheets were confirmed by TEM and corresponding SAED characterization. As shown in Figure 2a, the ED patterns were recorded from three different areas by aligning the electron beam perpendicular to the face of the sheet. These ED patterns manifest the same spot matrix and identical lattice orientation. On the basis of the indexing and the comparison with the theoretical crystal structure visualization of the (111) lattice plane in zinc blende CdTe (Supplementary Figure S3A), the CdTe nanosheets were formed by highly 3-D-oriented attachment of CdTe CQDs and exhibited quasi-sc features. Furthermore, this simple growth process can enable larger OA growth. Figure $2 \mathrm{~b}$ shows the TEM and SAED patterns of a typical CdTe nanobelt (3-4 $\mu \mathrm{m}$ scale). The ED patterns are consistent with the patterns in Figure $2 \mathrm{a}$, maintaining the quasi-sc feature dominated by the $\{111\}$ facets. The CdTe nanobelt with an arbitrary bending shape (see Supplementary Figure S4) also has a quasi-sc feature, which further confirms the flexibility of this technique. The SEM spatial morphologies of the as-grown CdTe nanobelts and nanosheets in Figures $2 \mathrm{c}$ and d clearly present the QDs matrix.
The 3-D and 2-D AFM representations of typical CdTe nanosheets and nanobelts grown on ITO conductive glass substrates are illustrated in Figures $2 \mathrm{e}$ and $\mathrm{f}$ and Supplementary Figure S5. From Figure 2e and $\mathrm{f}$, it is clear that the CdTe nanosheets have good contact with the ITO film. This good contact comes from the electrostatic forces arising from $\mathrm{Cd}^{2+}$ passivation and thiol capping on the nanosheet surface. The associated height profiles further reveal that the as-formed CdTe nanosheets or nanobelts have a rough surface with a thickness of $30 \sim 50 \mathrm{~nm}$, which is consistent with the SEM images in Figures $2 \mathrm{c}$ and $\mathrm{d}$. The good 2-D contact with the substrate and the quasi-sc feature of the CdTe nanosheets and nanobelts will be useful for single nanosheet/nanobelt-based electric devices. ${ }^{16}$ Figure $2 \mathrm{~g}$ shows the $\mathrm{CdTe}$ nanosheets in a high yield without any isolated nanoparticle impurities. To confirm good contact with the substrate, the I-V curves of an as-prepared CdTe nanobelt on $\mathrm{Si}$ wafer were obtained by CP-AFM measurements, as shown in Figure $2 \mathrm{~h}$. The unchanged linear parts around zero voltage in the I-V curve collected from different positions verify uniform resistance and good contact with the substrate. By contrast, the colloidal CdTe nanobelt with similar size and shape without in-situ growth on a silicon wafer has a larger resistance $\left(10^{3}\right.$ times larger) and is easy to fall off under the AFM tip connecting, as shown in Supplementary Figure S6. In comparison with colloidal CdTe, CdS nanosheets dropped on ITO substrates, as-grown CdTe, CdS nanosheets films have better contact with the ITO substrate, as confirmed by Supplementary Figures S7-S10. Verified by SEM morphology (Supplementary Figures S7) and I-V curve measurements (Supplementary Figures S9), due to poorer contact with ITO and worse overlap between nanosheets, the vertical resistance of dropped colloidal nanosheet films on ITO is $10^{3}$ times larger than as-grown nanosheet films on ITO.

Figure 3 presents the OA growth-formed morphology and crystallographic characterization of $c$ - $\mathrm{Ag}_{2-\delta}$ Te nanosheets and quasi-sc CdTe nanosheets. The SEM image in Figure 3a confirms the high purity of original nanosheet shapes without isolated nanoparticles. From Figures $3 \mathrm{~b}-\mathrm{f}$, although the $c-\mathrm{Ag}_{2-\delta} \mathrm{Te}$ nanosheets are polycrystalline, the shortrange multiple domains with continuous lattice fringe (Figures $3 \mathrm{~b}$ and $d$ ) and the HRTEM and SAED patterns (Figures 3e and f) confirm that they are formed by 3-D OA growth of $\mathrm{Ag}_{2-\delta} \mathrm{Te}$ nanocrystals. ${ }^{9}$ Consistent with Figures $2 \mathrm{a}$ and $\mathrm{b}$ analysis, the long-range multiple domains with continuous lattice fringe (Figure 3g, Supplementary 

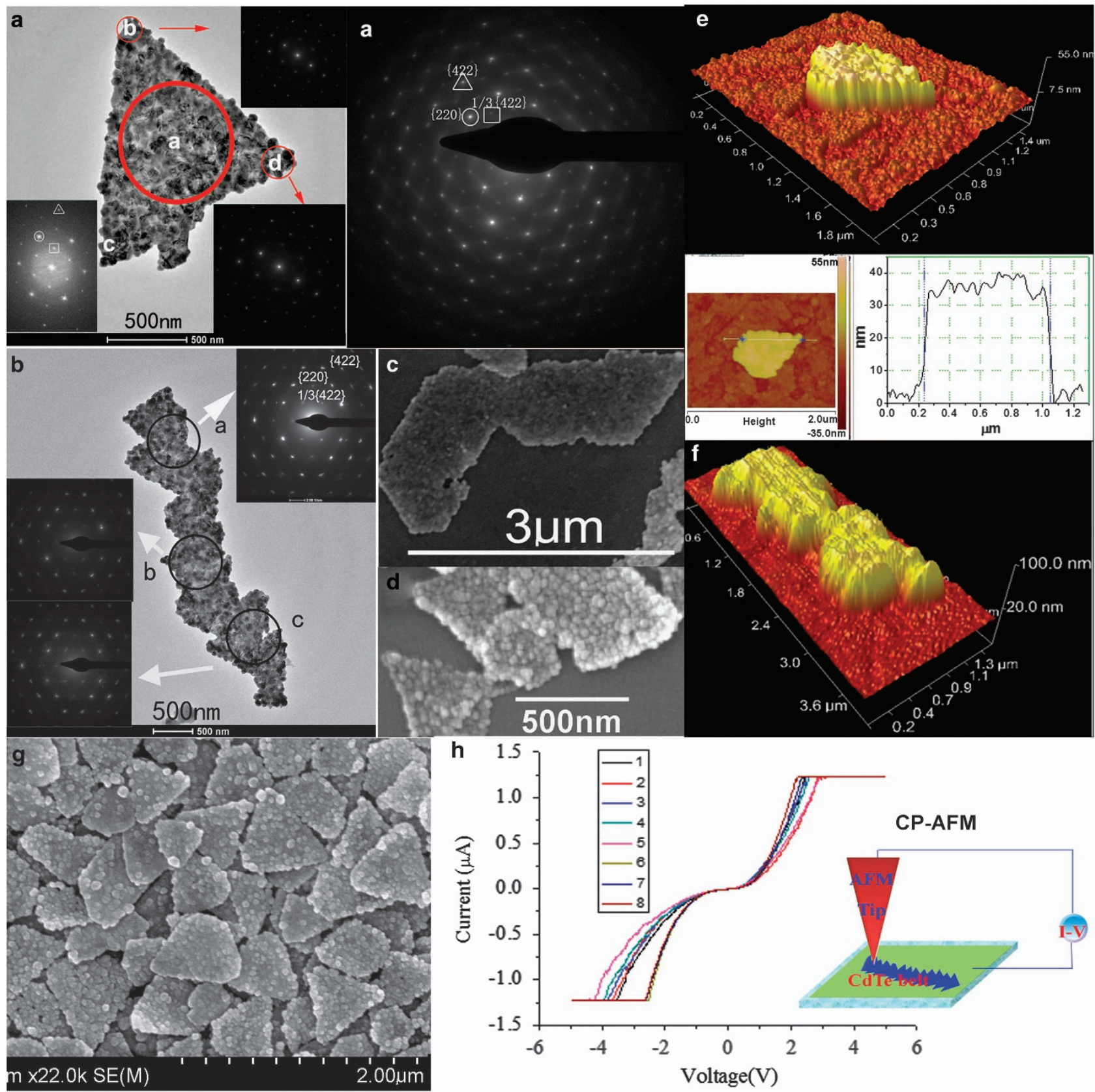

Figure 2 TEM, ED, AFM and SEM characterization of quasi-Sc CdTe nanosheets and nanobelts. (a) LRTEM image and ED patterns of selected areas (red circles, $a, b, d$ ) of a one micrometer-sized CdTe nanosheet; $c$ is the FFT pattern of the HRTEM image. In a and c, the circled strongest spots could be indexed as the $\{220\}$ reflections; the outer triangled spots with weak intensity could be assigned to the $\{422\}$ reflections; and the inner boxed spots corresponded to the formally forbidden 1/3\{422\} reflections. (b) TEM image and ED patterns of selected areas (black circles, a, b, c) of a CdTe nanobelt. (c, d) SEM images of a CdTe nanobelt and a CdTe nanosheet, respectively. (e, f) 3D and 2D representations of a CdTe nanosheet and a CdTe nanobelt on ITO conductive glass substrates measured by AFM. (g) SEM image of CdTe nanosheets in a high yield. (h) I-V curves of a CdTe nanobelt on silicon wafer by inserted CP-AFM measurement. Eight positions on the nanobelt were contacted with AFM tip to obtain I-V curves.

Figure S11) and the large-area SAED patterns (Figure 3i) confirm that, through this strategy, CdTe nanocrystals attach to each other with high orientation to become quasi-sc CdTe nanosheets. Figures $4 \mathrm{~A}$ and $\mathrm{B}$ display the CdS nanosheets and nanobelts $(6-7 \mu \mathrm{m})$ through this process. The SAED patterns (taken from three areas $\mathrm{a}, \mathrm{b}$ and $\mathrm{c}$ ) and the HRTEM analysis (Supplementary Figure S12) confirm the quasi-sc feature clearly, dominated by $\{001\}$ facets with a [001] growth direction. These reflection assignments agree well with the theoretical crystal structure visualization of the (002) lattice plane in wurtzite CdS
(Supplementary Figure S3B). The SEM image of the as-prepared CdS nanosheets on ITO glass in Supplementary Figure S13 also illustrates the CQD formation and good contact with the ITO film.

The amorphous state of $\mathrm{Ag}_{2-\delta} \mathrm{X}$ is essential for the quasi-sc state of the $\mathrm{CdX}$ nanosheets at micrometer sizes. In contrast to the reported cation exchange at quantum size by the Alivisatos group, ${ }^{25}$ if $\mathrm{Ag}_{2-\delta} \mathrm{Te}$ is modulated to be crystalline, the ensuing cation exchange reaction will generate polycrystalline CdTe nanosheets (see Supplementary Figure S14). The amorphous state of $\mathrm{Ag}_{2-\delta} \mathrm{X}$ promoted the motion of 


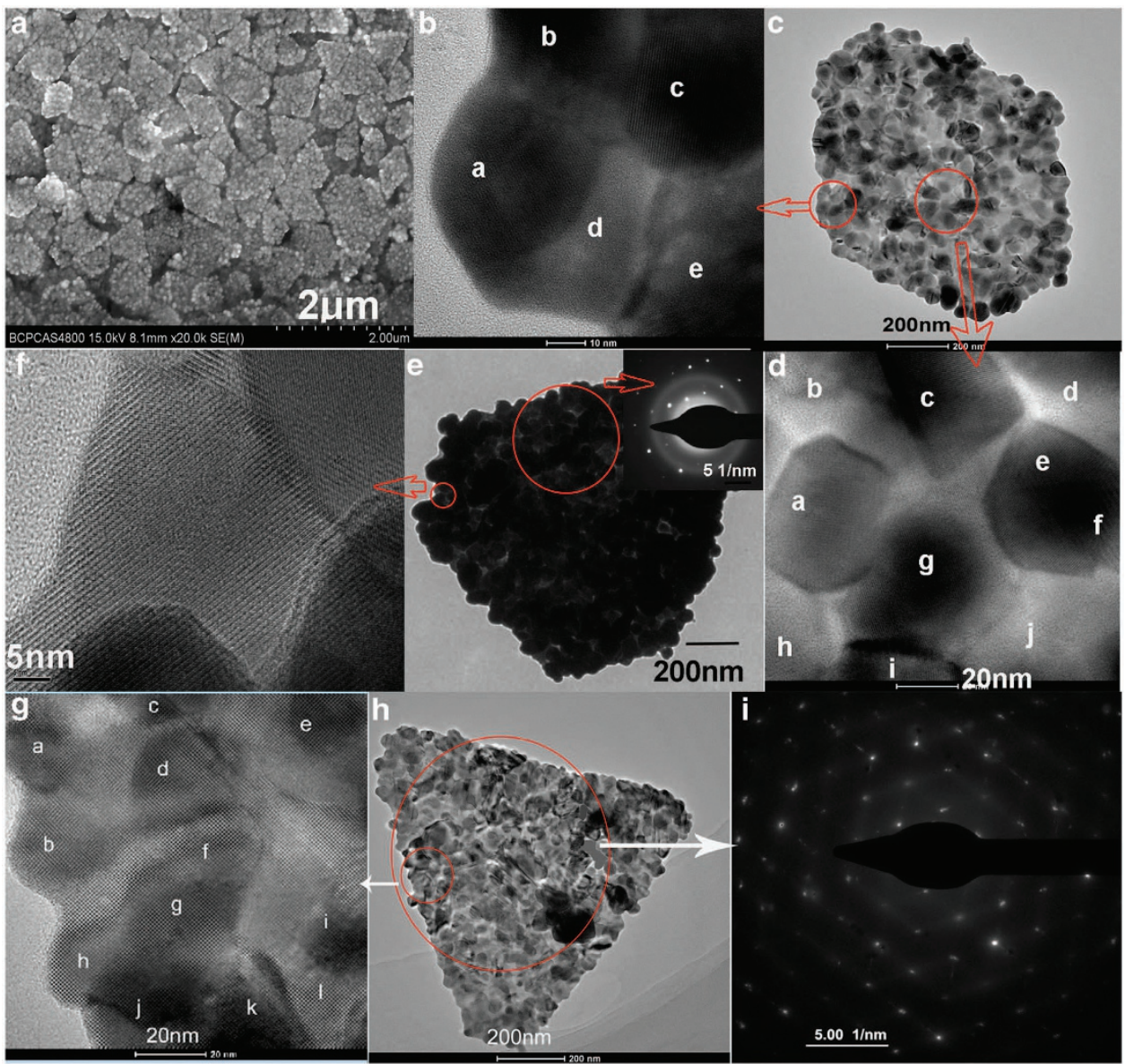

Figure 3 Morphology and crystallographic characterizations of $c-\mathrm{Ag}_{2-\delta} \mathrm{Te}$ nanosheets (a-f) and quasi-sc CdTe nanosheet (g-i): (a) SEM morphology of high yield $c-\mathrm{Ag}_{2-\delta} \mathrm{Te}$ nanosheets. (b-d) HRTEM images of the alignment of crystal orientation between different nanocrystal domains. Inserted a, b, c, d and so on letters are used to highlight the short-range multiple domains with clear and continuous lattice fringe. (e, f) SAED pattern and HRTEM of multiple domains with continuous lattice fringe of a $c-\mathrm{Ag}_{2-\delta} T e$ nanosheet. $(\mathbf{g}, \mathbf{h})$ HRTEM images of the alignment of crystal orientation between different nanocrystal domains. Inserted $\mathrm{a}, \mathrm{b}, \mathrm{c}, \mathrm{d}$ and so on letters are used to highlight the long-range multiple domains with clear and continuous lattice fringe. (i) SAED pattern of quasi-sc CdTe nanosheet.

cations as well as the growth of the quasi-sc domain of CdX due to a reduction of the grain boundary energies. As shown in Supplementary Figure S15, from the amorphous state to crystalline state, after the in-situ topotactic reaction on substrates, the shape, size and crystallization of $c-\mathrm{Ag}_{2-\delta} \mathrm{Te}$ or $\mathrm{CdX}$ CQD domains in the nanosheets changed. This observation means that, on the given rigid/flexible substrates, the introduction of the strong surface adsorption of dodecanethiol ligands and inorganic ions, such as $\mathrm{Cd}^{2+}$, facilitates the coalescence of CQDs by pure in-situ OA growth to yield nanosheets. The rotation and alignment of the as-formed CQD domains eliminate high surface energy facets and interfaces between them, ${ }^{26,27}$ resulting in their coalescence into quasi-sc nanosheets with lower surface energy facets exposed, as shown in Figure 4C. The polycrystalline state of $\mathrm{Ag}_{2-\delta} \mathrm{X}$ limits the following cation exchange reaction to rotate and align the CQD domains towards one growth direction due to different entropy-driven thermodynamics. ${ }^{27}$ The $a-\mathrm{Ag}_{2-\delta} \mathrm{X}$ nanoparticles form into nanosheets in this way and then become crystalline at $50-70^{\circ} \mathrm{C}$. Furthermore, with the coexistence of the strong van der Waals force from dodecanethiol and the electrostatic force from cations, ${ }^{28,29}$ the interfacial exothermal cation exchange reactions between the $a-\mathrm{Ag}_{2-\delta} \mathrm{X}$ nanosheets on the substrate and $\mathrm{Cd}^{2+}$ ions in solution promote the highly oriented 3-D OA growth of CQD domains into quasi-sc nanosheets on rigid/flexible substrates. Significantly, consistent with the enhanced electronic transport by inorganic atomic passivation on CQDs, ${ }^{1}$ the strong adsorption of cations after cation exchange provides effective contact with the electronegative substrates and between nanosheets (see also Figure 1). Without the substrate confinement and trace dodecanethiol addition, after these topotactic reactions, the free-standing Ag nanosheets will accordingly be converted to colloidal $\mathrm{Ag}_{2-\delta} \mathrm{X}$ nanoparticles and CdX CQDs (Supplementary Figure S16A). The Ag nanosheets are smooth with a good $s c$ feature at micrometer size (Supplementary Figure S16B), and the as-produced CdTe exists as $\sim 4-6 \mathrm{~nm}$ sized fluorescent CQDs (Supplementary Figure S16C). If insufficient dodecanethiol is added, the original nanosheet shape cannot be well maintained (Supplementary Figure S16D).

\section{XPS depth profile analysis}

Depth profiling allows us to determine the Ag and organic ligand residue distribution in the inner part of the as-prepared nanosheet film. ${ }^{30,31}$ For sputter depth profiling, Ar ions of $4 \mathrm{keV}$ and a scan size of $2 \mathrm{~mm} \times 2 \mathrm{~mm}$ were performed. As Oswald and Bruckner ${ }^{30}$ illustrated, these conditions could result in a typical sputter rate of 3-5 $\mathrm{nm} \mathrm{min}{ }^{-1}$ in as-prepared CdX nanosheet films. As shown in Figures $5 \mathrm{a}$ and $\mathrm{b}$, the atomic concentrations of $\mathrm{Cd}, \mathrm{S}$ and $\mathrm{Ag}$ are maintained at the approximate ratio of $\sim 55.4$ : 43.1 : 1.5 in the CdS 

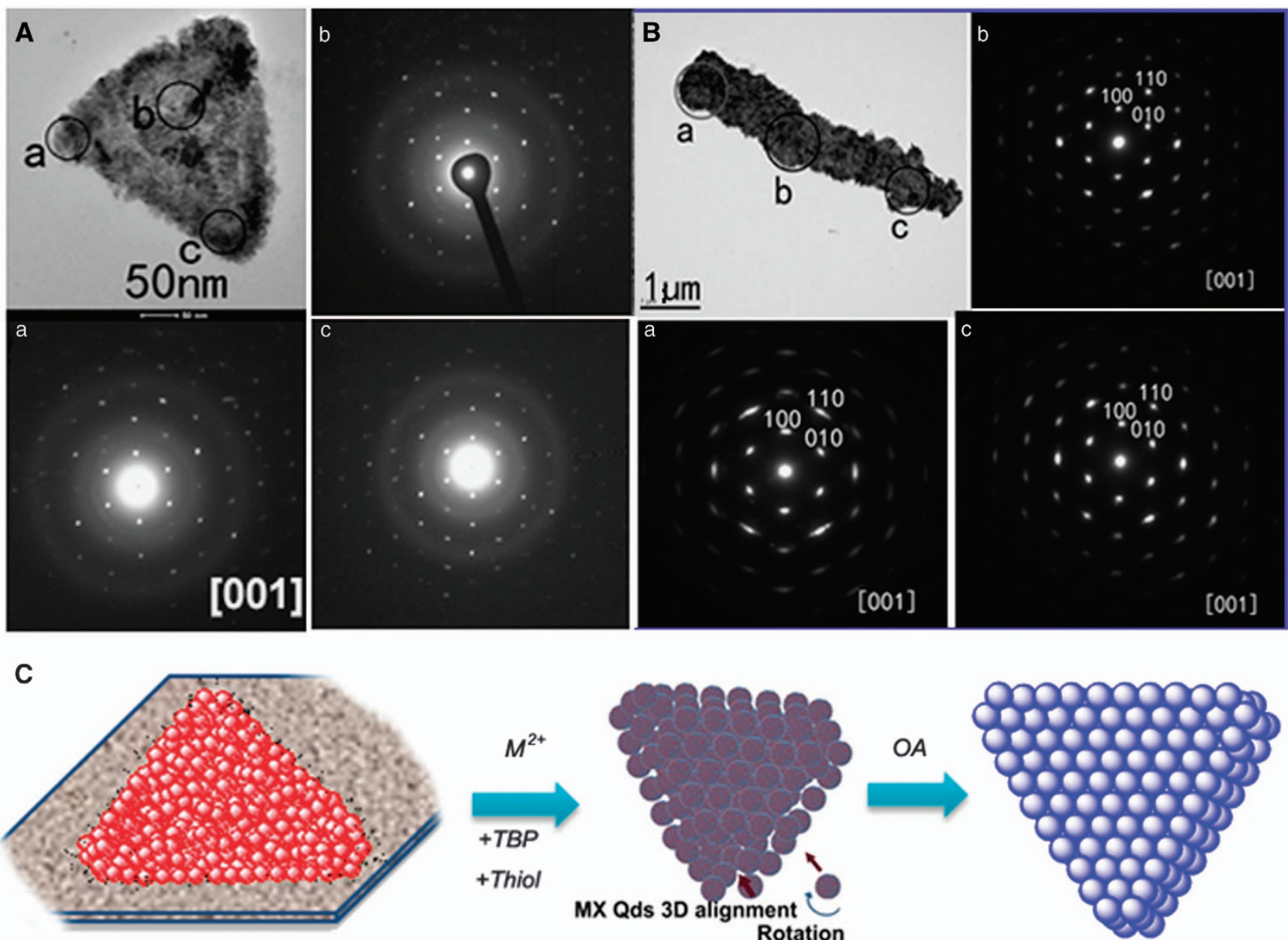

a- $A g_{2-8} \times N P$ aggregation

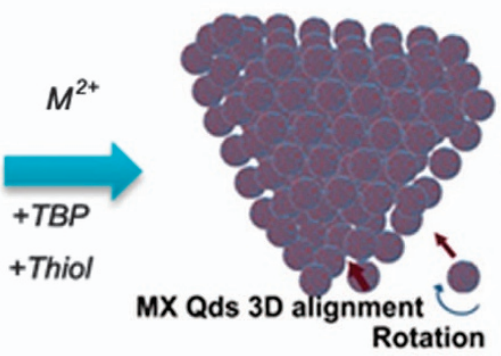

Rotation

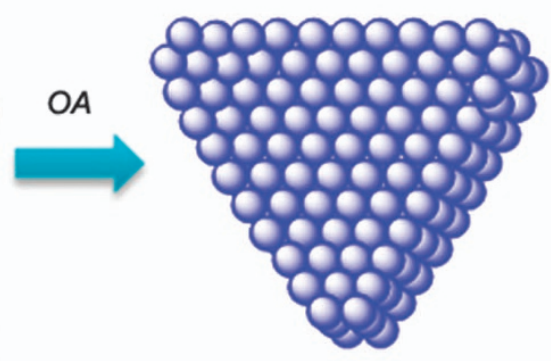

quasi-SC MX:Ag nanosheets

Figure 4 (A, B) TEM, ED characterizations (A, B) of quasi-sc CdS nanosheets and nanobelts. (C) OA growth mechanism of quasi-sc MX nanosheets with substrate confinement and thiol ligand capping.

nanosheet film as the sputtering proceeds into the film. However, the organic ligand-related C element decreases markedly from $65 \%$ to near zero as the sputter time increases. Figure $5 \mathrm{c}$ displays the $\mathrm{C} 1 \mathrm{~s}$ spectra evolution during depth profiling, from which the organic ligand residue on the nanosheet surface can be identified. On the surface of the nanosheet film (sputter time: 0 ), there is mainly C-C bonding $(284.8 \mathrm{eV})$ and $\mathrm{C}-\mathrm{O}$ bonding $(288.1 \mathrm{eV})$, which can be attributed primarily to the absorbed carbon and PVP capping ligands. ${ }^{32,33}$ As the sputter time increases (sputter time: 3, 6, 9 min), there is almost no C1s signal, which means there is nearly no organic ligand residue between the nanosheets inside the films. As shown in Figures $5 \mathrm{~d}$ and $\mathrm{e}$, the observed binding energy values of $\mathrm{Cd}_{3 \mathrm{~d}}$ and $\mathrm{S}_{2 \mathrm{p}}$ are in agreement with the reported data on CdS Qds. ${ }^{34-36}$ Furthermore, their invariant features and positions during the sputtering process confirm that the $\mathrm{Cd}$ and $\mathrm{S}$ elements maintain an unchanged chemical state from the surface into the film. In particular, as shown in Figure 5f, during depth profiling, the $\mathrm{Ag}$ residue is uniform throughout the film with a ratio of $\sim 1.5 \%$. The fitting results of the $\mathrm{Ag}_{3 \mathrm{~d} 5 / 2}$ and $\mathrm{Ag}_{3 \mathrm{~d} 3 / 2}$ peaks also confirm that the $\mathrm{Ag}$ residue exists as a $\mathrm{Ag}^{+}$dopant instead of $\mathrm{Ag}^{0}{ }^{36}$ From the EDS elemental mapping and line scan analysis of one single CdS nanosheet (Figures $5 \mathrm{~g}$ and $\mathrm{h}$ ), the Ag residue distributes uniformly across the nanosheet with a ratio of $\sim 2 \%$. In other words, through this topotactic reaction process, the Ag residue acts as an efficient dopant throughout the as-prepared CdX nanosheets. Enabled by the accompanying Ag doping, this strategy provides a new potential way to obtain enhanced carrier mobility and $p / n$ type control. This unprecedented strategy breaks the deadlock of cation exchange from the colloidal nanoparticle scale to bulk size and synchronously achieves efficient $\mathrm{Ag}$ doping in $\mathrm{CdX}$ films. ${ }^{25}$ It therefore offers the potential for site-fixed growth, complex compositional tailoring and metal ion doping of semiconductor nanosheets/nanobelts on substrates for electrical applications.

Wafer scale packing of as-prepared nanosheets on rigid/flexible substrates

When the evaporation rate of the solvent is controlled, the colloidal Ag sheets can be hierarchically packed on rigid/flexible substrates with good adhesion between each other and to substrates across a large scale (see Supplementary Figure S17). ${ }^{37}$ On the basis of this 3-D compact packing, the in-situ topotactic conversion here ensures the as-prepared $c$ - $\mathrm{Ag}_{2-\delta} \mathrm{X}$ and quasi-sc $\mathrm{CdX}$ nanosheets have large-scale overlap contact, although there are still many gaps in the film, as with the case of the CdTe nanosheets shown in Figure 6a. The cross-section image of the CdTe film in Figure 6a further confirms the vertical compact packing of $\mathrm{CdTe}$ nanosheets. Figure $6 \mathrm{~b}$ demonstrates the digital photographs of centimeter-scaled CdS, CdTe and $c-\mathrm{Ag}_{2-\delta} \mathrm{Te}$ 


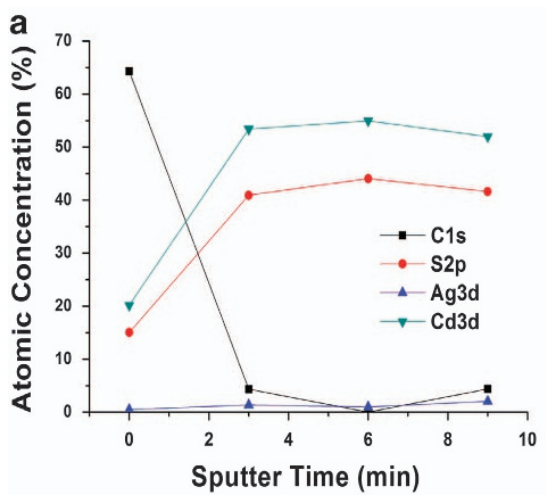

d

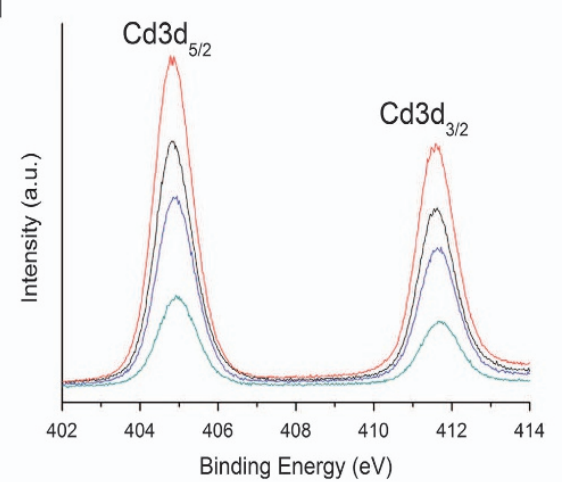

b

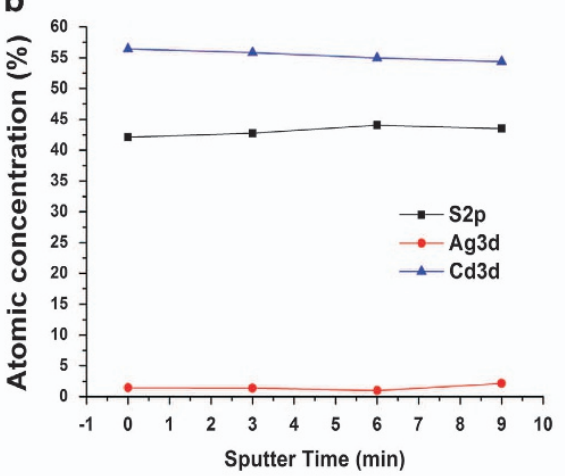

e

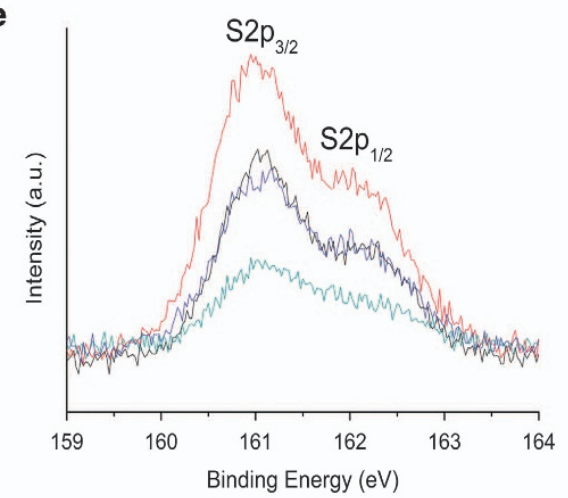

C

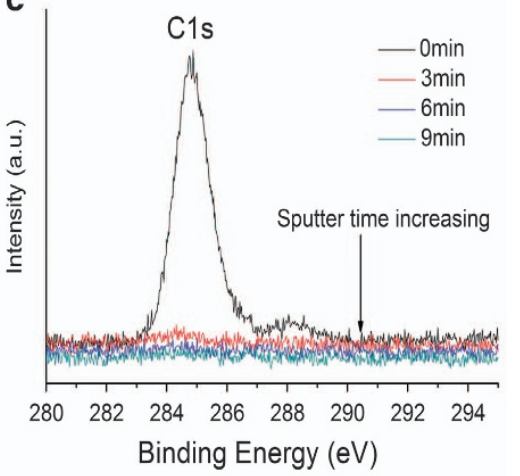

f

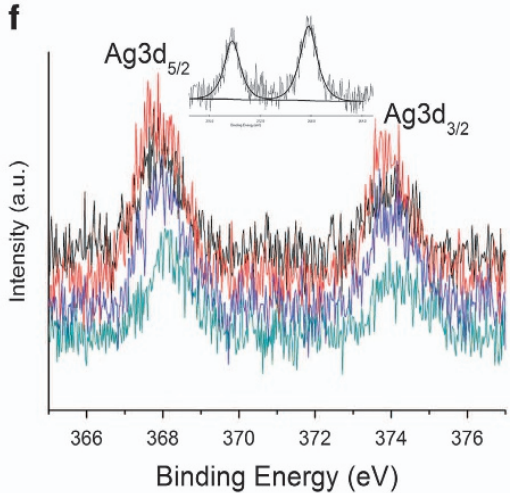

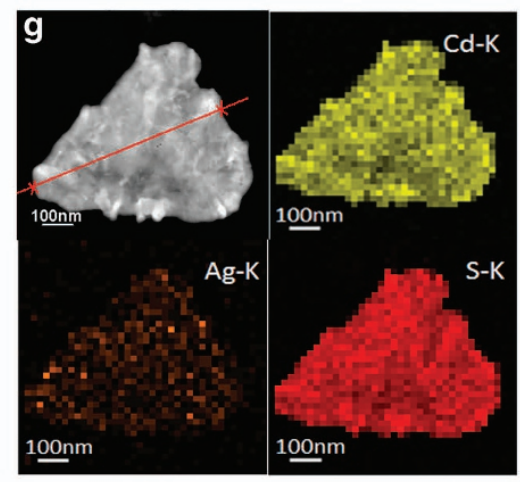

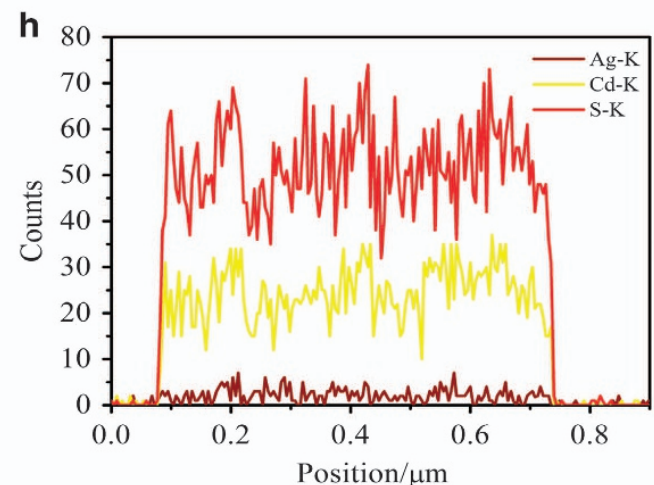

Figure $5(\mathbf{a}, \mathbf{b})$ The atomic percentage evolution of the $\mathrm{C}, \mathrm{Cd}, \mathrm{S}$ and $\mathrm{Ag}$ elements in as-prepared CdS nanosheet films by XPS depth profile analysis. (c-f) XPS spectra evolution of C1s, Cd3d, S2p and Ag3d during depth profiling. (g, h) Energy Dispersive Spectrum (EDS) elemental mapping and line scan of a CdS: Ag nanosheet. The $\mathrm{Cd}-\mathrm{K}, \mathrm{Ag}-\mathrm{K}$ and $\mathrm{S}-\mathrm{K}$ signals are presented in yellow, wine and red colors in Figure $5 \mathrm{~g}$.

films on rigid/flexible conductive substrates by this oriented assembly. For this reason, as shown in Figure 6c, there is only one noteworthy peak in the XRD pattern of the CdS and CdTe nanosheet films produced by hierarchical assembly ([002] peak for the CdS film and [111] peak for the CdTe film). Without this oriented assembly, the peaks of wurtzite CdS and zinc blende CdTe will arise gradually (see other patterns in Supplementary Figures S18A and B). As shown in Figure $6 \mathrm{~d}$, a close packed $c-\mathrm{Ag}_{2-\delta} \mathrm{Te}$ nanosheet film with a monoclinic phase can be prepared. The absence of organic ligands between nanosheets, the cation passivation and their oriented overlap assembly will ensure more efficient photo-induced electron/hole separation and transport in electronic devices. ${ }^{1,38,39}$

Enhanced electronic properties of wafer-scale $c$ - $\mathrm{Ag}_{2-\delta} \mathrm{Te}$ nanosheet films

The presence of organic ligands on the surface of CQDs strongly limits their technological applications where charge carrier transfer/transport has an important role. ${ }^{1}$ The as-prepared films here dispel this problem. A $1 \times 1 \mathrm{~cm}^{2}$ sized film composed of close-packed $c-\mathrm{Ag}_{2-\delta} \mathrm{Te}$ nanosheets has equal resistivities and linear I-V features along four sides, as shown in Supplementary Figure S19. In addition to the overlap contact, the $\mathrm{Cd}^{2+}$ exchange with most of the long-chain organic ligands is apparent. In addition, with orient-attached continuous single-crystallization between domains in the nanosheets, all these prerequisites ensure the efficient charge carrier transfer/transport between nanosheets. Without such large-scale uniform assembly, it is not easy to achieve this prerequisite. The Hall effect measurement of the film could then be performed. The electrical parameters of the asprepared films have accordingly been obtained and are shown in Figure 7 and Supplementary Figure S20. It is well known that nonstoichiometric $\mathrm{Ag}_{2 \pm} \delta$ Te has a potential magnetoresistance (MR) property due to its highly disordered stoichiometry. ${ }^{40,41}$ From Figures $7 \mathrm{a}$ and $\mathrm{b}$, the Hall voltage sensitivity, $\Delta \mathrm{V}_{\mathrm{H}}(\mathrm{T}, \mathrm{H}) / \mathrm{V}_{\mathrm{H}}(\mathrm{T}, 0)$, 

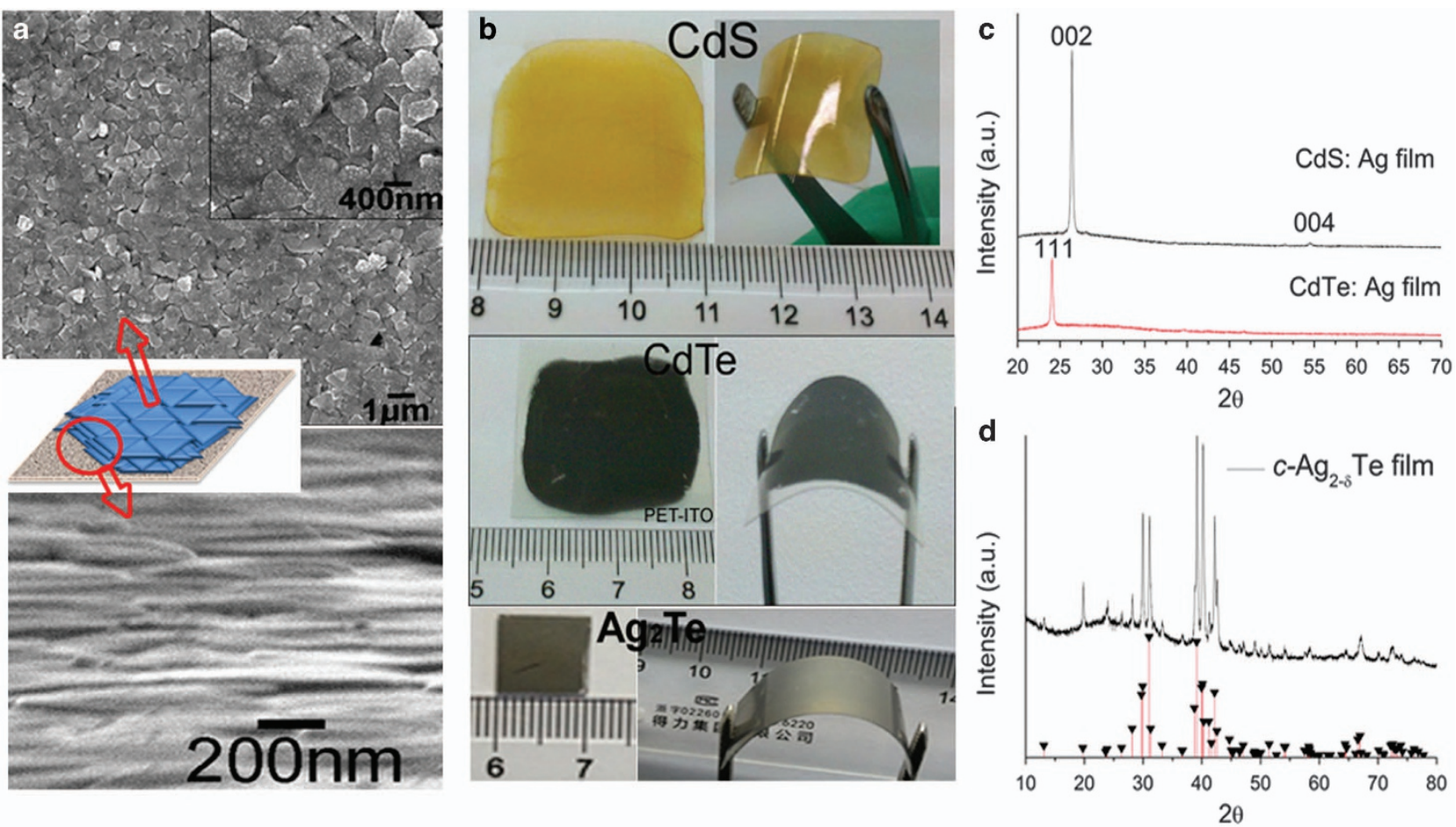

Figure 6 (a) SEM images of large-scale oriented close-packed quasi-sc CdTe nanosheet films. (b) Digital photographs of quasi-sc CdS, CdTe and c-Ag $2-\delta$ Te nanosheet films on rigid/flexible conductive substrates. (c) XRD patterns of CdS and CdTe nanosheet films with parallel and overlap assembly on substrates. (d) XRD patterns of $c-\mathrm{Ag}_{2-8} \mathrm{Te}$ films in Figure $\mathbf{b}$ (red solid lines, JCPDS \# 34-0142).
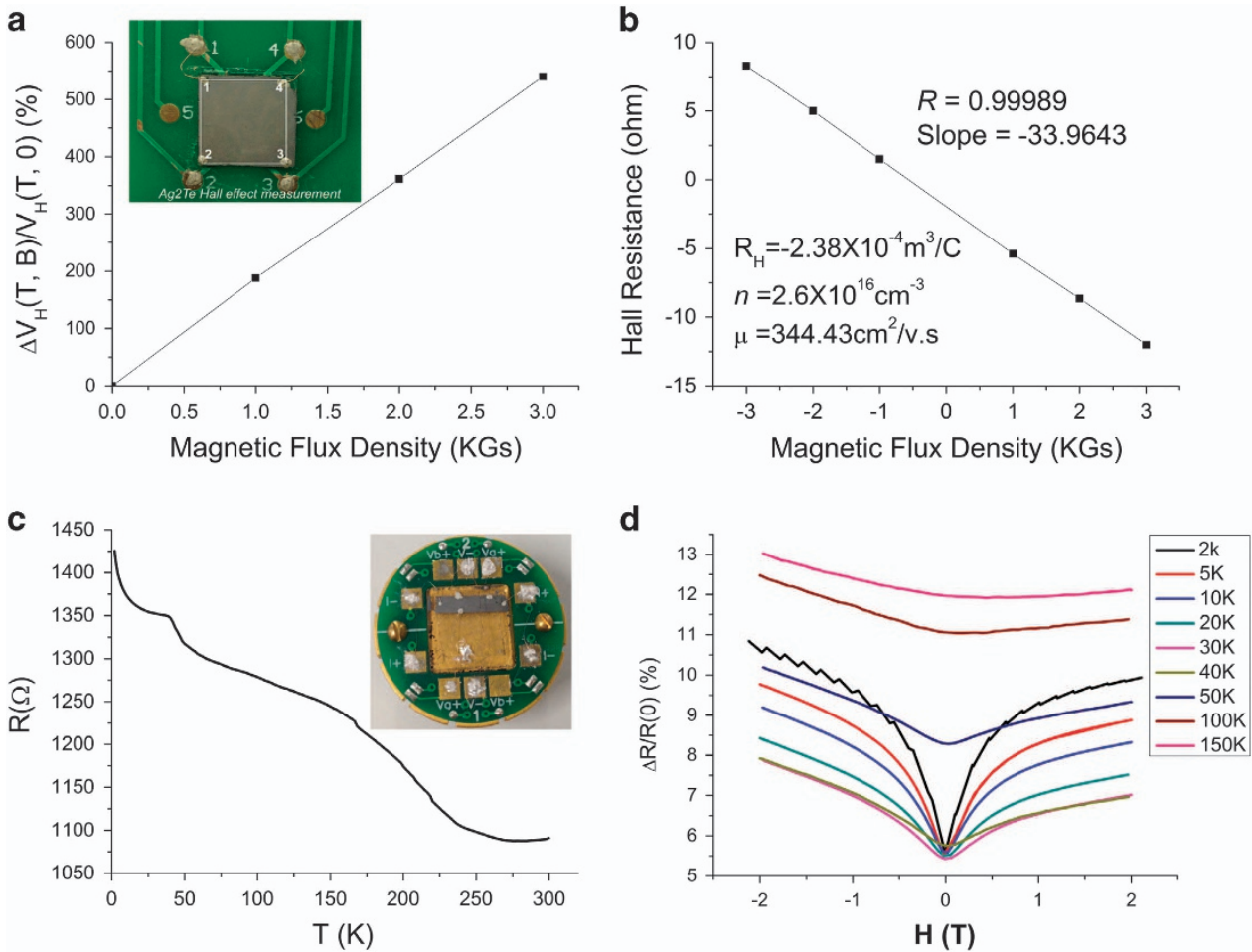

Figure 7 (a) Hall voltage sensitivity, $\Delta V_{H}(T, B) / V_{H}(T, 0)$, of $c-A_{2-\delta} T e$ nanosheet film to magnetic field at room temperature. Inset, digital photograph of Hall effect device, where the white lines highlight the square measurement area. (b) Field dependence of Hall resistance at room temperature. Inset, the correlation coefficient $R$ and slope of linear variation and the calculated electronic parameters, Hall coefficient $R_{\mathrm{H}}$, carrier concentration $n$ and carrier mobility $\mu$. (c) Resistance versus temperature for $c-\mathrm{Ag}_{2-\delta} \mathrm{Te}$ nanosheet film. The inset shows a digital image of the five-electrode Hall bar for transport measurement. (d) Magnetoresistance of $c-\mathrm{Ag}_{2-\delta} \mathrm{Te}$ nanosheet film in perpendicular magnetic field under different low temperatures (2-150 K). 
a

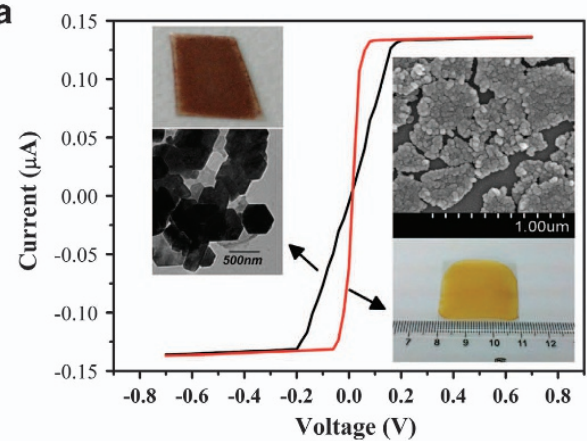

C

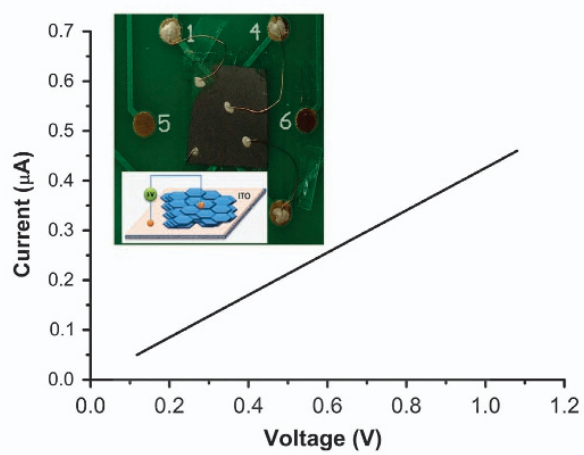

e

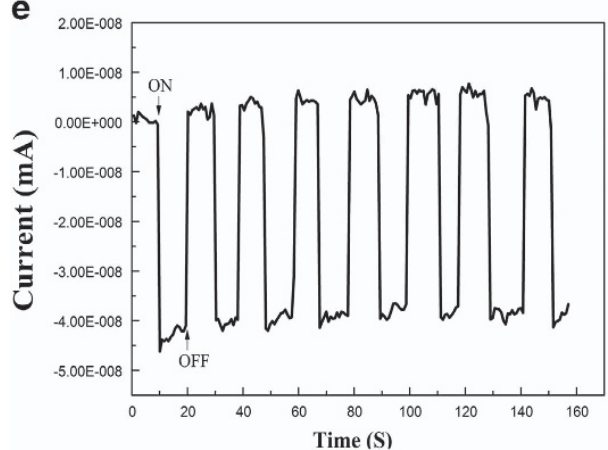

b

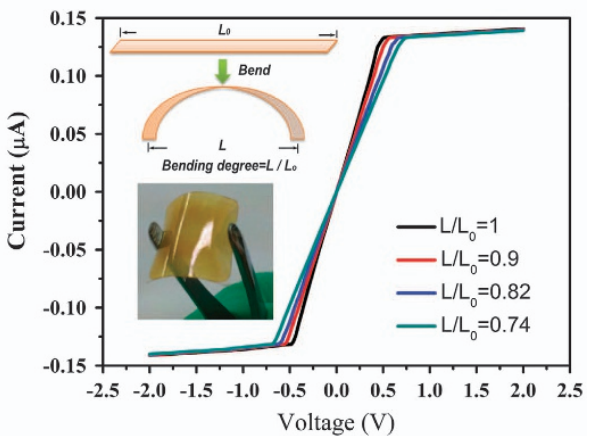

d
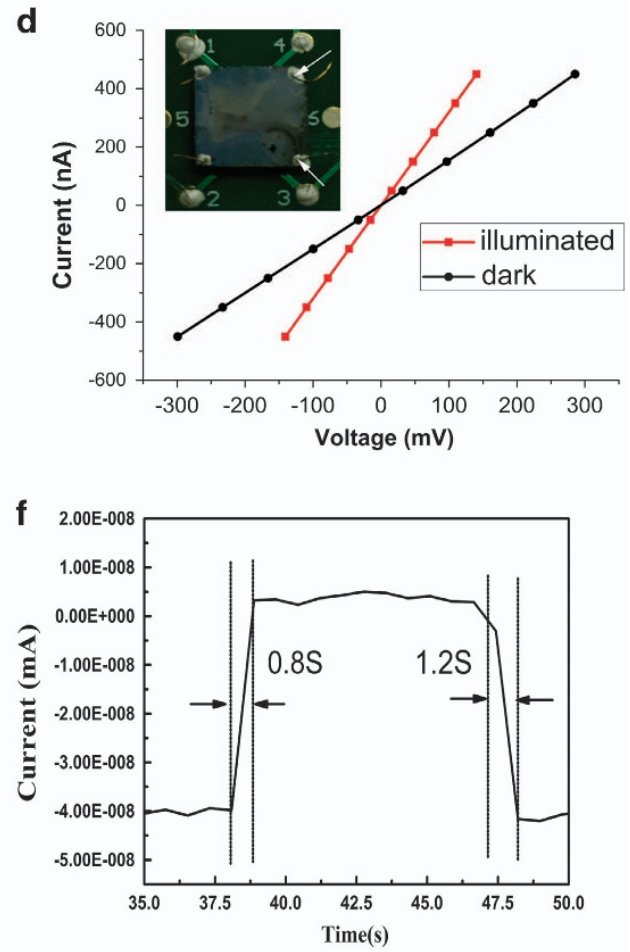

Figure 8 (a) I-V curves comparison of as-prepared CdS nanosheet film to colloidal single-crystalline CdS nanoplate formed film on PET conductive substrate. Insets are the TEM of single-crystalline CdS nanoplates, bulk film picture (left) and SEM of as-prepared CdS nanosheet film, bulk film picture (right). (b) I-V curves of as-prepared CdS nanosheet film on flexible PET substrate with different degree of bending force (L/Lo). Insets are the schematic bending degree and picture of bending. (c) Typical I-V curve of Ag electrode/CdTe nanosheet film/PET conductive substrate. Inset shows a digital photo and a cartoon of the measurement device. (d) Typical I-V curves of CdTe nanosheet film with and without illumination by $300 \mathrm{~W}$ Xe lamp. Inset is a digital photo of the device for lateral I-V measurement. (e) Photoconductivity response of bulk-sized CdTe nanosheet film on exposure to on/off white light at room temperature without bias voltage. (f) Response time and recovery time of photodetector.

of the obtained film to magnetic field $\mathrm{H}$ is enhanced up to $188 \%$ at room temperature, demonstrating a potential Hall effect sensor application. ${ }^{42}$ The Hall resistance varies linearly with the magnetic flux density with a correlation coefficient $R$ of 0.99989 . The Hall coefficient $R_{\mathrm{H}}$ is calculated to be $-2.38 \times 10^{-4} \mathrm{~m}^{3} \mathrm{C}^{-1}$ when the film thickness $d$ is $7 \mu \mathrm{m}$. The negative $R_{\mathrm{H}}$ indicates $n$-type electronic impurities in the as-prepared $c-\mathrm{Ag}_{2-\delta} \mathrm{Te}$ film. ${ }^{43}$ The carrier concentration $n\left(n=\frac{1}{\left|R_{H}\right| e}\right)$ is calculated to be $2.6 \times 10^{16} \mathrm{~cm}^{-3}$. The carrier mobility $\mu\left(\mu=R_{\mathrm{H}} / \rho\right.$, where $\rho$ is the resistivity) is $344.43 \mathrm{~cm}^{2} \mathrm{v}^{-1}$ $\mathrm{s}^{-1}$. Although it is smaller than the reported value for a single $\mathrm{Ag}_{2} \mathrm{Te}$ nanowire prepared by solid state sintering methods, ${ }^{44}$ this value is a distinct improvement compared with previous reports of colloidal $\mathrm{Ag}_{2 \pm \delta} \mathrm{Te}$ nanoparticle films. ${ }^{45}$ Five-electrode transport measurements were conducted on bulk-sized $c-\mathrm{Ag}_{2-\delta} \mathrm{Te}$ nanosheet assembled films using a physical property measurement system (PPMS). Five silver strips with good ohmic contact with the $c-\mathrm{Ag}_{2-\delta} \mathrm{Te}$ nanosheets were obtained easily by dip-coating, as shown in the inset in Figure 7c. The temperature dependence of the resistance for this type of film was measured between 2 and $300 \mathrm{~K}$ in zero field, as plotted in Figure 7c. The film exhibits an increase in resistance at zero field when the temperature decreases, which indicates that the bulk nature is nonmetallic. The observation of weak antilocalization phenomena, one important requirement for topological insulators (TL), has been reported at the nanometer scale for films of metal telluride by severe solid-state methods. ${ }^{46-49}$ Here, as shown in Figure 7d, we observed not only weak antilocalization phenomena in $c-\mathrm{Ag}_{2-\delta} \mathrm{Te}$ nanosheet assembled films but also MR quantum oscillation behavior at low temperatures. ${ }^{47}$ Therefore, this property is likely to secure the topological surface state and indicate the potential application for TL devices. $^{46-49}$ 
Enhanced electronic properties of wafer-scale CdX nanosheet films Owing to the close hierarchical assembly (Figure 6) and the quantum size effect arising from small but continuous single-crystalline domains in nanosheets, bulk-sized CdX nanosheet films also exhibit efficient charge carrier transfer/transport. Deduced from I-V curves in Figure 8a, compared with single-crystalline CdS nanoplate films formed by dip-coating, ${ }^{50}$ the as-prepared CdS nanosheet films have a smaller resistivity. In particular, as-prepared CdS nanosheet films on flexible PET substrates show mechanical stability when subjected to bending forces. As shown in Figure 8b, with different bending forces, the CdS nanosheet film shows almost invariable resistance that can be calculated from the linear part of I-V curves between -0.5 and $0.5 \mathrm{~V}$ (see also Supplementary Table S21). However, the single-crystalline CdS nanoplate film (see inset picture in Figure 8a) falls off of the PET substrate when given a bending force. As-prepared flexible $c-\mathrm{Ag}_{2-\delta} \mathrm{Te}$ nanosheet films also exhibit good mechanical stability, as shown in Supplementary Figure S22. The ohmic contacts between the electrode and film here can be obtained easily by the dip-coating of conductive silver glue on the film. The linear I-V conductivities of the film and film to substrate (Figures $8 \mathrm{c}$ and d, Supplementary Figure S23) illustrate the orient-attached continuous single-crystallization between domains and uniform packing of as-prepared nanosheets, which is necessary for efficient electron transport. Compared with CQD films formed by as-obtained colloidal CQDs (Supplementary Figures S16 A, $\mathrm{C}$ and S24) and reported nanoparticle films prepared by chemical bath deposition, ${ }^{51,52}$ the smaller resistivities of these nanosheet assembled films (see detailed analysis in S-25) indicate the synergetic effect of the good contact between the nanosheet and the substrate, the close contact of nanosheets, the absence of organic ligands between the nanosheets and the quantum size effect arising from small but continuous single-crystalline domains in nanosheets (see also quantum size effect on efficient charge carrier transfer/transport analysis in S-26). Although there are still many gaps between the nanosheets, their hierarchical overlap stacking and inorganic cation passivation ensure efficient electron transport. The ultraviolet-visible and infrared (UV-VIS-NIR) absorption spectra (Shimadzu Corporation, Japan) of the as-prepared CdTe films in Supplementary Figure S27 show efficient whole-solar-spectrum absorption $(300-950 \mathrm{~nm})$. The above characteristics indicate their potential photovoltaic applications. The distinct conductivity changes with or without white light illumination (Figure 8d, Supplementary Figure S28) exhibit potential photodetector applications. Figure $8 \mathrm{e}$ illustrates the potential photoresponse switching behavior of the as-prepared CdTe nanosheet films. It can be observed that the photocurrent can be reproducibly switched from the ON to the OFF state by periodically turning the Xe lamp on and off. The quick response $(0.8 \mathrm{~s})$ and recovery $(1.2 \mathrm{~s})$ times, as shown in Figure 8 f, indicate its ability to follow a quickly varying optical signal.

\section{CONCLUSIONS}

In summary, micrometer-sized non-stoichiometric $c-\mathrm{Ag}_{2-\delta} \mathrm{X}$, Ag-doped quasi-sc CdX nanosheets/nanobelts have been prepared successfully on rigid (ITO glass) and flexible (PET) substrates by a topotactic reaction enabling the OA of CQDs. This strategy extends the cation exchange technique from the CQD dimension to a bulksized film. By introducing the strong surface adsorption of cations and dodecanethiol ligands that can provide van der Waals forces and electrostatic forces, the OA of CQDs into micro-sized nanosheets/ nanobelts (up to $6-7 \mu \mathrm{m}$ ) on diverse substrates has been achieved. The in-situ hierarchical assembly of these nanosheets on substrates at a large scale effectuates enhanced electronic properties, such as Hall effect sensitivity, linear magnetoresistance and potential TL device applications of the $c-\mathrm{Ag}_{2-\delta} \mathrm{X}$ nanosheet films and the efficient electron transport and photoresponse properties of $\mathrm{CdX}$ nanosheet films. This sequential assembly spanning dimensions from CQDs to bulk film and the good mechanical stability of these flexible films provide an alternative bottom-up fabrication approach to nanotechnology for high-performance electronic and optoelectronic devices.

\section{CONFLICT OF INTEREST}

The authors declare no conflict of interest.

\section{ACKNOWLEDGEMENTS}

This work was supported by the National Natural Science Foundation (91123001, 21322105 and 51372025), the Research Fund for the Doctoral Program of Higher Education of China (2011101120016), Beijing Excellent Talent Training Project (2012D009011000007) and Program for New Century Excellent Talents in University (NCET-11-0793). We thank Dr K Chang of State Key Laboratory of Low-Dimensional Quantum Physics, Tsinghua University for PPMS measurements and helpful discussions; Professor GZ Shen and Dr Z Liu of State Key Laboratory for Superlattices and Microstructures, Institute of Semiconductors, CAS for I-V curves measurements under different bending force and helpful discussions; Professor C Wang and Dr M Xu of National Center for Nanoscience and Technology, China for CP-AFM measurements and helpful discussions.

Author contributions: H.-m.Q. and J.-t.Z. conceived and designed the experiments. H.-m.Q., Q.Z. and B.-s.D. performed the materials synthesis, and some of characterizations and measurements. L.G. and J.-x.Z. performed the AFM characterizations and results fitting. J.-t.Z. and H.-s.Z. supervised the project. All authors contributed to the scientific discussions.

1 Tang, J., Kemp, K. W., Hoogland, S., Jeong, K. S., Liu, H., Levina, L., Furukawa, M., Wang, X., Debnath, R., Cha, D., Chou, K., Fischer, A., Amassian, A., Asbury, J. B. \& Sargent, E. H. Colloidal-quantum-dot photovoltaics using atomic-ligand passivation. Nat. Mater. 10, 765-771 (2011).

2 Dong, A., Chen, J., Vora, P. M., Kikkawa, J. M. \& Murray, C. B. Binary nanocrystal superlattice membranes self-assembled at the liquid-air interface. Nature 466, 474-477 (2010).

3 Baker, J. L., Cooper, A.W., Toney, M. F., Geissler, P. L. \& Alivisatos, A. P. Device-scale perpendicular alignment of colloidal nanorods. Nano Lett. 10, 195-201 (2010).

4 Penn, R. L. \& Banfield, J. F. Imperfect oriented attachment: dislocation generation in defect-free nanocrystals. Science 281, 969-971 (1998).

5 Banfield, J. F., Welch, S. A., Zhang, H., Ebert, T. T. \& Penn, R. L. Aggregation-based crystal growth and microstructure development in natural iron oxyhydroxide biomineralization products. Science 289, 751-754 (2000).

6 Zhang, X., Zhang, J., Zhao, J., Pan, B., Kong, M., Chen, J. \& Xie, Y. Half-metallic ferromagnetism in synthetic $\mathrm{Co}_{9} \mathrm{Se}_{8}$ nanosheets with atomic thickness. J. Am. Chem. Soc. 134, 11908-11911 (2012).

7 Schliehe, C., Juarez, B. H., Pelletier, M., Jander, S., Greshnykh, D., Nagel, M., Meyer, A., Foerster, S., Kornowski, A., Klinke, C. \& Weller, H. Ultrathin PbS sheets by two-dimensional oriented attachment. Science 329, 550-553 (2010).

8 Tang, Z., Zhang, Z., Wang, Y., Glotzer, S. C. \& Kotov, N. A. Self-assembly of CdTe nanocrystals into free-floating sheets. Science 314, 274-278 (2006).

9 Zhang, Q., Liu, S. J. \& Yu, S. H. Recent advances in oriented attachment growth and synthesis of functional materials: concept, evidence, mechanism, and future. J. Mater. Chem. 19, 191-207 (2009).

10 Xiang, G., Li, T., Zhuang, J. \& Wang, X. Large-scale synthesis of metastable $\mathrm{TiO}_{2}(\mathrm{~B})$ nanosheets with atomic thickness and their photocatalytic properties. Chem. Comm. 46, 6801-6803 (2010).

11 Vaughn, D. D., In, S. \& Schaak, R. E. A precursor-limited nanoparticle coalescence pathway for tuning the thickness of taterally-uniform colloidal nanosheets: the case of SnSe. ACS Nano 5, 8852-8860 (2011).

12 Wang, F., Richards, V., Shields, S. \& Buhro, W. Kinetics and mechanisms of aggregative nanocrystal growth. Chem. Mater. 26, 5-21 (2014).

13 Chhowalla, M., Shin, H. S., Eda, G., Li, L., Loh, K. P. \& Zhang, H. The chemistry of two-dimensional layered transition metal dichalcogenide nanosheets. Nat. Chem. 5 263-275 (2013).

14 Wang, Q. H., Zadeh, K. K., Kis, A., Coleman, J. N. \& Strano, M. S. Electronics and optoelectronics of two-dimensional transition metal dichalcogenides. Nat. Nanotech. 7, 699-712 (2012).

15 Xue, D., Tan, J., Hu, J., Hu, W., Guo, Y. \& Wan, L. Anisotropic photoresponse properties of single micrometer-sized GeSe nanosheet. Adv. Mater. 24, 4528-4533 (2012). 
16 Zhang, J., Li, D., Chen, R. \& Xiong, Q. H. Laser cooling of a semiconductor by 40 kelvin. Nature 493, 504-508 (2013).

17 Chen, O., Zhao, J., Chauhan, V. P., Cui, J., Wong, C., Harris, D. K., Wei, H., Han, H. S., Fukumura, D., Jain, R. K. \& Bawendi, M. G. Compact high-quality CdSe-CdS core-shell nanocrystals with narrow emission linewidths and suppressed blinking. Nat. Mater. 12, 445-451 (2013).

18 Talapin, D. V., Rogach, A. L., Kornowski, A., Haase, M. \& Weller, H. Highly luminescent monodisperse CdSe and CdSe/ZnS nanocrystals synthesized in a hexadecylaminetrioctylphosphine oxide - trioctylphospine mixture. Nano Lett. 1, 207-211 (2001).

19 Peng, X. G. An essay on synthetic chemistry of colloidal nanocrystals. Nano Res. 2 , 425-447 (2009).

20 Regulacio, M. D. \& Han, M. Y. Composition-tunable alloyed semiconductor nanocrystals. Acc. Chem. Res. 43, 621-630 (2010)

21 Smith, A. M. \& Nie, S. M. Semiconductor nanocrystals: structure, properties, and band gap engineering. Acc. Chem. Res. 43, 190-200 (2010).

22 Osada, M. \& Sasaki, T. Two-dimensional dielectric nanosheets: novel nanoelectronics from nanocrystal building blocks. Adv. Mater. 24, 210-228 (2012).

23 Zhou, J., Wang, J., Huang, Y., Liu, G., Wang, L., Chen, S., Li, X., Wang, D., Song, Y. \& Jiang, L. Large-area crack-free single-crystal photonic crystals via combined effects of polymerization-assisted assembly and flexible substrate. NPG Asia Mater. 4 e21 (2012)

24 Zhang, J. T., Tang, Y., Lee, K. \& Ouyang, M. Nonepitaxial growth of hybrid core-shell nanostructures with large lattice mismatches. Science 327, 1634-1638 (2010).

25 Son, D. H., Hughes, S. M., Yin, Y. D. \& Alivisatos, A. P. Cation exchange reactions in ionic nanocrystals. Science 306, 1009-1012 (2004).

26 Wang, Z., Schliehe, C., Wang, T., Nagaoka, Y., Cao, Y. C., Bassett, W. A., Wu, H., Fan, H. Y. \& Weller, H. Deviatoric stress driven formation of large single-crystal PbS nanosheet from nnanoparticles and in situ monitoring of oriented attachment. J. Am. Chem. Soc. 133, 14484-14487 (2011).

27 Zhang, J., Huang, F. \& Lin, Z. Progress of nanocrystalline growth kinetics based on oriented attachment. Nanoscale 2, 18-34 (2010)

28 Bai, F., Wang, D., Huo, Z., Chen, W., Liu, L., Liang, X., Chen, C., Wang, X., Peng, Q. \& $\mathrm{Li}, \mathrm{Y}$. D. A versatile bottom-up assembly approach to colloidal spheres from nanocrystals. Angew. Chem. Int. Ed. 46, 6650-6656 (2007).

29 Brinker, C. J., Lu, Y. F., Sellinger, A. \& Fan, H. Y. Evaporation-induced self-assembly: nanostructures made easy. Adv. Mater. 11, 580-585 (1999).

30 Oswald, S. \& Bruckner, W. XPS depth profile analysis of non-stoichiometric NiO films. Surf. Interface Anal. 36, 17-22 (2004).

31 Claflin, B., Leedy, K. D. \& Look, D. C. Dopant profiles in heavily doped ZnO. Opt. Eng. 52, 053801-1-4 (2013).

$32 \mathrm{Mao}, \mathrm{H}$., Feng, J., Ma, X., Wu, C. \& Zhao, X. One-dimensional silver nanowires synthesized by self-seeding polyol process. J. Nanopart. Res. 14, 887-1-15 (2012)

33 Zhang, J., Cao, C., Lv, Q., Li, C. \& Zhu, H. The preparation and characterization of CNx film with high nitrogen content by cathode electrodeposition. Appl. Surf. Sci. 214, 364-369 (2003).

34 Peng, X. G., Schlamp, M. C., Kadavanich, A. V. \& Alivisatos, A. P. Epitaxial growth of highly luminescent $\mathrm{CdSe} / \mathrm{CdS}$ core/shell nanocrystals with photostability and electronic accessibility. J. Am. Chem. Soc. 119, 7019-7029 (1997).

35 Pan, D., Wang, Q., Pang, J., Jiang, S., Ji, X. L. \& An, L. Semiconductor 'nano-onions' with multifold alternating $\mathrm{CdS} / \mathrm{CdSe}$ or $\mathrm{CdSe} / \mathrm{CdS}$ structure. J. Chem. Mater. 18, 4253-4258 (2006).

36 Lin, L., Wen, Y. Q., Liang, Y., Zhang, N. \& Xiao, D. Aqueous synthesis of $\mathrm{Ag}^{+}$doped CdS quantum dots and its application in $\mathrm{H}_{2} \mathrm{O}_{2}$ sensing. Anal. Methods 5, 457-464 (2013).

37 Zhang, J. T., Li, X. L., Sun, X. M. \& Li, Y. D. Surface enhanced Raman scattering effects of silver colloids with different shapes. J. Phys. Chem. B 109, 12544-12548 (2005).
38 Wang, Y., Liu, J., Tran, H. D., Mecklenburg, M., Guan, X. N., Stieg, A. Z., Regan, B. C., Martin, D. C. \& Kaner, R. B. Morphological and dimensional control via hierarchical assembly of doped oligoaniline single crystals. J. Am. Chem. Soc. 134, 9251-9262 (2012).

39 Wang, Y., Tang, Z., Podsiadlo, P., Elkasabi, Y., Lahann, J. \& Kotov, N. A. Mirror-like photoconductive layer-by-layer thin films of Te nanowires: the fusion of semiconductor, metal, and insulator properties. Adv. Mater. 18, 518-522 (2006).

40 Wang, X. L., Du, Y., Dou, S. X. \& Zhang, C. Room temperature giant and linear magnetoresistance in topological insulator $\mathrm{Bi}_{2} \mathrm{Te}_{3}$ nanosheets. Phys. Rev. Lett. 108, 266806-1-5 (2012)

41 Xu, R., Husmann, A., Rosenbaum, T. F., Saboungi, M. L., Enderby, J. E. \& Littlewood, P. B. Large magnetoresistance in non-magnetic silver chalcogenides. Nature 390, 57-60 (1997).

42 Lu, H., Sandvik, P., Vertiatchikh, A., Tucker, J. \& Elasser, A. High temperature Hall effect sensors based on AIGaN/GaN heterojunctions. J. Appl. Phys. 99, 114510-1-4 (2006).

43 Ahn, K., Li, C., Uher, C. \& Kanatzidis, M. Improvement in the thermoelectric figure of merit by La/Ag cosubstitution in PbTe. Chem. Mater. 21, 1361-1367 (2009).

44 Lee, S., In, J., Yoo, Y., Jo, Y., Park, Y. C., Kim, H., Koo, H. C., Kim, J., Kim, B. \& Wang, K. L. Single Crystalline $\beta-\mathrm{Ag}_{2} \mathrm{Te}$ Nanowire as a New Topological Insulator. Nano Lett. 12, 4194-4199 (2012).

45 Cadavid, D., Ibáňez, M., Shavel, A., Durá, O. J., Torre, M. A. L. \& Cabot, A. Organic ligand displacement by metal salts to enhance nanoparticle functionality: thermoelectric properties of Ag $\mathrm{Te}$. J. Mater. Chem. A 1, 4864-4870 (2013).

46 Liu, M., Zhang, J., Chang, C., Zhang, Z., Feng, X., Li, K., He, K., Wang, L., Chen, X., Dai, X., Fang, Z., Xue, Q. K., Ma, X. \& Wang, Y. Crossover between weak antilocalization and weak localization in a magnetically doped topological insulator. Phys. Rev. Lett. 108, 036805-1-5 (2012).

47 Ning, W., Du, H., Kong, F., Yang, J., Han, Y., Tian, M. \& Zhang, Y. One-dimensional weak antilocalization in single-crystal $\mathrm{Bi}_{2} \mathrm{Te}_{3}$ nanowires. Sci. Rep. 3, 1564-1-6 (2013).

48 Cha, J. J., Kong, D., Hong, S. S., Analytis, J. G., Lai, K. \& Cui, Y. Weak antilocalization in $\mathrm{Bi}_{2}\left(\mathrm{Se}_{\mathrm{x}} \mathrm{Te}_{1-\mathrm{x}}\right)_{3}$ nanoribbons and nanoplates. Nano Lett. 12, 1107-1111 (2012).

49 Lang, M., He, L., Xiu, F., Yu, X., Tang, J., Wang, Y., Kou, X., Jiang, W., Fedorov, A. \& Wang, K. Revelation of topological surface states in $\mathrm{Bi}_{2} \mathrm{Se}_{3}$ thin films by in situ $\mathrm{Al}$ passivation. ACS Nano 6, 295-302 (2012).

50 Wang, J., Feng, H., Chen, K. \& You, T. Seeded-synthesis and characterization of CdS hexagonal nanoplates. Mater. Lett. 104, 87-90 (2013).

51 Metin, H. \& Esen, R. Annealing studies on CBD grown CdS thin films. J. Cryst. Growth. 258, 141-148 (2003)

52 Ikhmayies, S. J. \& Ahmad-Bitar, R. N. Characterization of vacuum evaporated CdTe thin films prepared at ambient temperature. Mater. Sci. in Semi. Proc. 16 , $118-125$ (2013).

(c) (i)(3) (2) This work is licensed under a Creative Commons BY NG SA Attribution-NonCommercial-ShareAlike 4.0 International License. The images or other third party material in this article are included in the article's Creative Commons license, unless indicated otherwise in the credit line; if the material is not included under the Creative Commons license, users will need to obtain permission from the license holder to reproduce the material. To view a copy of this license, visit http://creativecommons.org/licenses/ by-nc-sa/4.0/

Supplementary Information accompanies the paper on the NPG Asia Materials website (http://www.nature.com/am) 